\title{
Population implications of lipid lowering for prevention of coronary heart disease: data from the 1995 Scottish health survey
}

\author{
I U Haq, L E Ramsay, E J Wallis, C G Isles, L D Ritchie, P R Jackson
}

\begin{abstract}
Objective-To determine the proportion of the population, firstly, with cholesterol $\geqslant 5.0 \mathrm{mmol} / 1$ and, secondly, with any cholesterol concentration, who might benefit from statin treatment for the following: secondary prevention of coronary heart disease (CHD); primary prevention at CHD risk $30 \%, 20 \%, 15 \%$, and $6 \%$ over 10 years; and primary prevention at projected CHD risk $20 \%$ over 10 years (CHD risk at age 60 years if actual age $<60$ years).

Subjects-Random stratified sample of 3963 subjects aged 35-64 years from the Scottish health survey 1995.

Results-For secondary prevention $7.8 \%$ (95\% confidence interval (CI) $6.9 \%$ to $8.6 \%$ ) of the population with cholesterol $\geqslant 5.0 \mathrm{mmol} / 1$ would benefit from statins. For primary prevention, the prevalence of people at CHD risk 30\%, 20\%, 15\%, and $6 \%$ over 10 years is $1.5 \%(95 \%$ CI $1.2 \%$ to $1.9 \%$ ), $5.4 \%$ (95\% CI $4.7 \%$ to $6.1 \%$ ), $9.7 \%$ (95\% CI $8.8 \%$ to $10.6 \%$ ), and $32.9 \%$ (95\% CI $31.5 \%$ to $34.4 \%$ ), respectively. At projected CHD risk $20 \%$ over 10 years, $12.4 \%$ (95\% CI $11.4 \%$ to $13.5 \%$ ) would be treated with statins. Removing the $5.0 \mathrm{mmol} / \mathrm{l}$ cholesterol threshold makes little difference to population prevalence at high CHD risk.

Conclusions-Statin treatment would be required for $7.8 \%$ of the population for secondary prevention. For primary prevention, among other factors, guidelines should take into account the number of patients needing treatment at different levels of CHD risk when choosing the CHD risk to target. The analysis supports a policy of targeting treatment at CHD risk $30 \%$ over 10 years as a minimum, as recommended in current British guidelines, with a move to treating at CHD risk $15 \%$ over 10 years as resources permit.

(Heart 2001;86:289-295)
\end{abstract}

Keywords: statins, coronary risk, secondary prevention, primary prevention

Section of Clinical Pharmacology and Therapeutics, Department of Medicine and Pharmacology, Royal Hallamshire Hospital, Sheffield S10 2JF, UK I U Haq

L E Ramsay

E J Wallis

P R Jackson

Department of

Medicine, Dumfries and Galloway Royal Infirmary, Bankend

Road, Dumfries DG1 4AP, UK

C G Isles

Department of General Practice and

Primary Care, University of

Aberdeen, Forester

Hill Health Centre,

Westburn Road,

Aberdeen AB25 2AY,

UK

L D Ritchie

Correspondence to: Professor Ramsay

D.Colley@Sheffield.ac.uk

Accepted 9 May 2001
The results of five randomised trials have established that cholesterol lowering by statins prevents coronary heart disease (CHD) in both secondary ${ }^{1-3}$ and primary prevention, ${ }^{45}$ with cholesterol reduction of $20-25 \%$ reducing major coronary events by about $33 \%$. Additional benefits are prevention of hospital admissions, revascularisation procedures, and, in secondary but not primary prevention, strokes. In these trials relative risk reduction for major CHD events by statins was constant at about 33\%, both between trials and in subgroup analyses within trials. This holds true for primary and secondary prevention, in men or women, and in numerous other subgroups such as patients with diabetes or hypertension, or smokers. Because relative risk reduction is constant, benefit from statin treatment is determined by the absolute CHD risk of an individual person, ${ }^{6}$ not by pretreatment concentrations of cholesterol or other lipid fractions. ${ }^{78}$ Absolute CHD risk determines the number of patients who need to be treated to prevent one major coronary event ${ }^{9}$; the cost effectiveness of treatment ${ }^{7}{ }^{10}$; the proportion of the population that would require treatment ${ }^{7}$; and the total cost of statin treatment. ${ }^{7}{ }^{10}$ Given unlimited resources, statin treatment can be justified at a CHD event risk as low as $6 \%$ over 10 years ${ }^{5}$ but current guidelines all advise a risk threshold higher than this. ${ }^{11-14}$ All emphasise secondary prevention as the first priority, but for primary prevention they differ in their recommendations for the CHD risk to target. Joint British Society guidelines recommend treatment at CHD risk $30 \%$ over 10 years as a minimum and treatment at $15 \%$ over 10 years as optimal. ${ }^{11}$ Joint European Task Force guidelines suggest treatment at CHD risk $20 \%$ over 10 years and when CHD risk projected to the age of 60 years is estimated at $\geqslant 20 \%$ over 10 years in those younger than 60 years old. ${ }^{12}$

We have previously set out the implications of possible policies for statin treatment for the English population given the evidence then. The analysis of the Scottish health survey population described here ${ }^{15}$ has important differences. The Scottish health survey database has additional information, for example on peripheral vascular disease, which allows more complete quantification of the need for secondary prevention, and on high density lipoprotein (HDL) cholesterol, which notably increases the accuracy of $\mathrm{CHD}$ risk estimation for primary prevention. ${ }^{16}{ }^{17}$ The previous analysis did not consider treatment at CHD risk below $15 \%$ over 10 years in accordance with the evidence then available. ${ }^{7}$ Here we examine the implications of statin treatment at CHD risk $6 \%$ over 10 years and at CHD risk $20 \%$ over 10 years projected to the age of 60 years as recommended in the joint European guidelines. The previous analysis was based on a total cholesterol threshold of $5.5 \mathrm{mmol} / \mathrm{l}$ but guidelines 
now advise treatment at a cholesterol threshold of $5.0 \mathrm{mmol} / 1$ because of new evidence. ${ }^{11-14}$ However, this threshold is based on a subgroup analysis of one trial, ${ }^{2}$ and it remains possible that statins should be prescribed for all those with high CHD risk regardless of the total cholesterol concentration. We have examined the implications in the population of applying the $5.0 \mathrm{mmol} / 1$ cholesterol threshold, or of adopting no threshold at all and treating all people at high CHD risk.

\section{Methods}

SCOTTISH HEALTH SURVEY 1995

This survey, commissioned by the Scottish Office Department of Health and carried out by the Joint Health Surveys Unit at Social and Community Planning Research and University College London Medical School, examined CHD risk factors in the Scottish population aged 16-64 years and was conducted between March 1995 and February 1996. ${ }^{15}$ A random sample of addresses selected by postcode was visited by an interviewer, who randomly selected one resident aged between 16 and 64 years, and elicited information on symptoms, diagnoses, and treatment of cardiovascular disease. In subjects who agreed, a nurse subsequently obtained details of prescribed medications and measured blood pressure and total and HDL cholesterol. Blood was drawn with minimal venous stasis and cholesterol concentration was measured in a single laboratory by the cholesterol oxidase method using a SUMMIT monitor (American Monitor Co, now disbanded, USA).

DEFINITION OF RISK FACTORS

Myocardial infarction, angina, stroke, and diabetes were defined in the survey and this paper as recall of a doctor's diagnosis of these conditions. Peripheral vascular disease was diagnosed by a symptom questionnaire for claudication. Smoking history was recorded and we define as smokers those who smoke cigarettes currently or in the past year, as in the

A

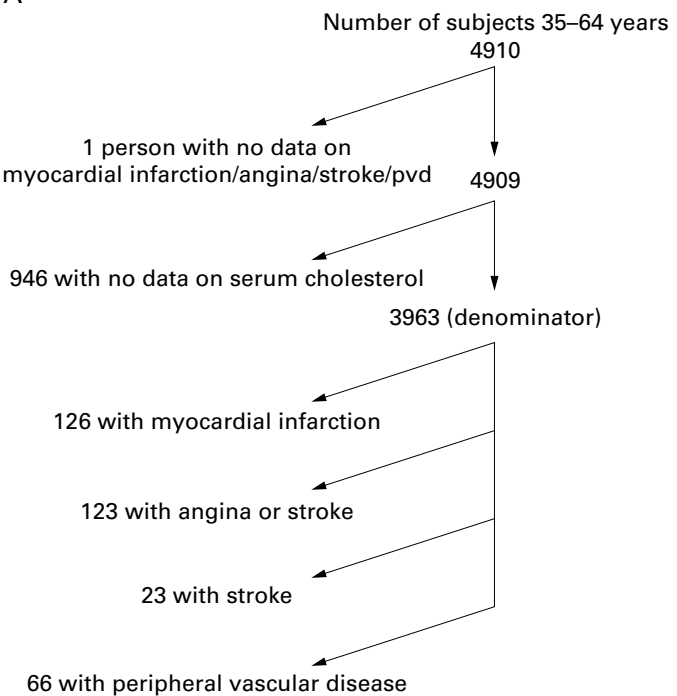

Framingham study. ${ }^{18}$ Blood pressure was measured three times at one minute intervals after sitting for five minutes, using a Dinamap 8100 monitor (GE Medical Systems IT, Basingstoke, UK) and an appropriate cuff size. The mean of the second and third readings was analysed.

\section{LEVELS OF CHD RISK EXAMINED FOR PRIMARY} PREVENTION

Four CHD event rates were examined for those with cholesterol $\geqslant 5.0 \mathrm{mmol} / 1$ and separately for those with any cholesterol concentration, as follows:

- CHD risk 30\% over 10 years, which was recommended for treatment in Standing Medical Advisory Committee guidance ${ }^{13}$ and as a minimum in recent Joint British Societies guidelines. ${ }^{11}$

- $C H D$ risk $20 \%$ over 10 years, at which treatment is recommended in the Second Joint European Task Force guidelines. ${ }^{12}$ Treatment is also recommended at a "projected risk" of $20 \%$ over 10 years, meaning the CHD risk assuming that age is 60 years if age is below 60, or actual age if age is $\geqslant 60$ years.

- $C H D$ risk $15 \%$ over 10 years, which is considered optimal in Joint British guidelines ${ }^{11}$ - a goal to be attained progressively after treatment of CHD risk $30 \%$ over 10 years has been implemented.

- $C H D$ risk 6\% over 10 years, which was the level of risk of placebo treated people in the AFCAPS/TexCAPS (Air Force/Texas coronary atherosclerosis prevention study). ${ }^{5}$ This analysis therefore explores the implications of treating for primary prevention all who have a risk of CHD similar to that in the AFCAPS/TexCAPS study, which represents the limits of current trial evidence.

METHOD OF ANALYSIS

The analysis in 4910 subjects aged 35-64 years is outlined in figs 1 and 2. One subject had no

Figure 1 Sequential route of data analysis from the Scottish health survey to estimate the proportion of the population for secondary prevention. (A) Subjects with any serum total cholesterol concentration. (B) Subjects with serum total cholesterol concentration $\geqslant 5.0 \mathrm{mmoll}$. 
A
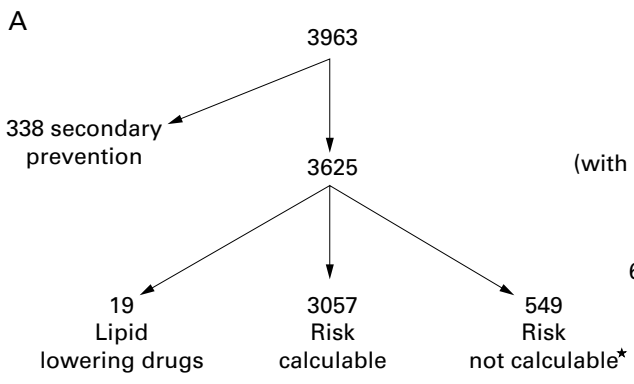

B

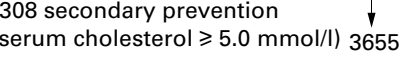

Figure 2 Sequential route of data analysis from the Scottish health survey to estimate the proportion of the population for primary prevention at different levels of CHD event risk. (A) Subjects with any serum total cholesterol concentration. (B) Subjects with serum total cholesterol concentration $\geqslant 5.0$ mmol/l. *Risk was not calculable for 549 subjects (or 454 subjects with serum cholesterol $\geqslant 5.0 \mathrm{mmol} / \mathrm{l}$ ) because of absence of data for one or more coronary risk factors.

data on myocardial infarction, angina, stroke, or peripheral vascular disease, and 946 had no cholesterol measurement, leaving 3963 with full data on myocardial infarction, angina, stroke, peripheral vascular disease, and serum cholesterol. Data were analysed in those with serum cholesterol $\geqslant 5.0 \mathrm{mmol} / \mathrm{l}$ and in all subjects regardless of any cholesterol threshold. The latter analysis included 665 subjects $(16.8 \%)$ who had serum total cholesterol $<5.0 \mathrm{mmol} / \mathrm{l}$.

\section{SECONDARY PREVENTION}

Among the 3298 (83.2\%) subjects who had serum cholesterol $\geqslant 5.0 \mathrm{mmol} / \mathrm{l}$, those with a history of myocardial infarction were identified; then those with angina but no myocardial infarction; then those with stroke but no myocardial infarction or angina; and finally those with peripheral vascular disease but no myocardial infarction, angina, or stroke. The denominator for estimates of prevalence in the population was 3963 subjects - that is, all those aged 35-64 years with information on these diagnoses and a serum cholesterol measurement (fig 1). Prevalence and 95\% confidence intervals (CIs) were calculated for 10 year age bands. This analysis was repeated for all subjects regardless of the cholesterol concentration, using the same denominator.

Table 1 Coronary heart disease risk factors and prevalence of myocardial infarction, angina, stroke, and peripheral vascular disease in people aged 35-64 years who did and did not have serum cholesterol measurement

\begin{tabular}{|c|c|c|c|c|}
\hline & \multicolumn{2}{|l|}{ Men } & \multicolumn{2}{|l|}{ Women } \\
\hline & $\begin{array}{l}\text { Cholesterol } \\
\text { measured }\end{array}$ & $\begin{array}{l}\text { Cholesterol not } \\
\text { measured }\end{array}$ & $\begin{array}{l}\text { Cholesterol } \\
\text { measured }\end{array}$ & $\begin{array}{l}\text { Cholesterol not } \\
\text { measured }\end{array}$ \\
\hline Serum cholesterol $(\mathrm{mmol} / \mathrm{l})$ & $6.1(0.03)$ & - & $6.0(0.03)$ & - \\
\hline Number & 1812 & 396 & 2151 & 550 \\
\hline Age (years) & $48.7(0.21)$ & $49.2(0.46)$ & $48.7(0.19)$ & $49.7(0.40)^{\star}$ \\
\hline Systolic blood pressure ( $\mathrm{mm} \mathrm{Hg}$ ) & $134.5(0.43)$ & $137.1(0.76)$ & $129.4(0.43)$ & $132.8(0.63)^{\star}$ \\
\hline Smokers (\%) & 35.4 & $46.2^{\star \star}$ & 36.0 & $44.7^{\star \star}$ \\
\hline Diabetes (\%) & 2.5 & 3.8 & 2.1 & $4.0^{\star}$ \\
\hline Lipid lowering drugs (\%) & 1.6 & 0.8 & 0.7 & 0.4 \\
\hline Myocardial infarction (\%) & 5.0 & 5.8 & 1.6 & 2.7 \\
\hline Angina $(\%)$ & 5.6 & 7.1 & 4.5 & 4.7 \\
\hline Stroke (\%) & 1.7 & 3.0 & 0.8 & 2.2 \\
\hline Peripheral vascular disease (\%) & 2.2 & 3.8 & 2.1 & 2.2 \\
\hline
\end{tabular}

Values are expressed as means (SEM).

Significance tests compare people of the same sex with and without cholesterol measured: ${ }^{\star} \mathrm{p}<0.05,{ }^{\star \star} \mathrm{p}<0.001$.
PRIMARY PREVENTION

To examine the prevalence of CHD risk strata in subjects free of vascular disease, those with myocardial infarction, angina, stroke, or peripheral vascular disease were excluded, leaving 3665 people who had cholesterol measurement but no indication for secondary prevention. Of these, 665 had serum total cholesterol $<5.0 \mathrm{mmol} / 1$ and 2990 cholesterol $\geqslant 5.0 \mathrm{mmol} / \mathrm{l}$ (fig 2). Nineteen were taking lipid lowering drugs and were excluded because CHD risk could not be calculated. There were complete data for 2517 (84\%) subjects to calculate CHD risk (see below), and 454 had one or more variables missing for risk calculation. Exclusion of these subjects might bias the findings, and the prevalence of risk level strata in the population was calculated assuming the same prevalence for those with missing data ( $\mathrm{n}=454)$ as in those in whom risk could be calculated ( $n=2517)$. The analysis was repeated for subjects with any cholesterol concentration - that is, retaining those with cholesterol $<5.0 \mathrm{mmol} / 1$. The method of analysis ensures that the population prevalence of those for primary prevention is additive to that for secondary prevention.

\section{CHD RISK CALCULATION}

Risk of CHD events was calculated by the Framingham risk function ${ }^{18}$ using the following risk factors: age, sex, systolic blood pressure, total to HDL cholesterol ratio, smoking status, and diabetes. Left ventricular hypertrophy was assumed to be absent. The Framingham function is accurate for prediction between 4-12 years, and risks of CHD events over five years were computed for consistency with previous analyses ${ }^{7}$ and because the duration of the statin trials has been approximately five years. British and European guidelines now express risk as $\mathrm{CHD}$ events in 10 years, and the calculated five year estimates were doubled and expressed as CHD risk over 10 years to conform with this. Comparison of 10 year risk calculated thus, and 10 year risk calculated directly by the Framingham risk function, shows no important difference. 
Table 2 Proportion (\%) of people at different ages with a history of myocardial infarction, angina, stroke, or peripheral vascular disease and serum total cholesterol $\geqslant 5.0 \mathrm{mmol} / \mathrm{l}$, and separately for those with any cholesterol concentration

\begin{tabular}{|c|c|c|c|c|c|c|c|c|c|c|c|c|}
\hline \multirow[b]{2}{*}{ Age (years) } & \multicolumn{6}{|l|}{ Men } & \multicolumn{6}{|l|}{ Women } \\
\hline & $\begin{array}{l}\text { No with TC } \\
\text { measured }\end{array}$ & $M I$ & Angina & Stroke & $P V D$ & Total & $\begin{array}{l}\text { No with TC } \\
\text { measured }\end{array}$ & $M I$ & Angina & Stroke & $P V D$ & Total \\
\hline \multicolumn{13}{|c|}{$\mathrm{TC} \geqslant 5.0 \mathrm{mmol} / 1$} \\
\hline $35-44$ & 671 & 1.0 & 0.0 & 0.0 & 0.9 & 1.9 & 804 & 0.1 & 0.9 & 0.4 & 0.6 & 2.0 \\
\hline $45-54$ & 583 & 3.6 & 2.1 & 0.5 & 0.7 & 6.9 & 673 & 1.9 & 1.6 & 0.3 & 2.5 & 6.4 \\
\hline $55-64$ & 558 & 9.5 & 5.6 & 2.0 & 2.5 & 19.5 & 674 & 2.7 & 7.7 & 0.4 & 2.1 & 12.9 \\
\hline Total 35-64 & 1812 & 4.5 & 2.4 & 0.8 & 1.3 & 8.9 & 2151 & 1.5 & 3.3 & 0.4 & 1.7 & 6.8 \\
\hline $95 \% \mathrm{CI}$ & & 3.5 to 5.4 & 1.7 to 3.1 & 0.4 to 1.2 & 0.8 to 1.9 & 7.6 to 10.3 & & 1.0 to 2.0 & 2.5 to 4.0 & 0.1 to 0.6 & 1.2 to 2.2 & 5.7 to 7.9 \\
\hline \multicolumn{13}{|l|}{ All TC } \\
\hline Total 35-64 & 1812 & 5.0 & 2.6 & 0.8 & 1.5 & 9.9 & 2151 & 1.6 & 3.5 & 0.4 & 1.8 & 7.3 \\
\hline $95 \% \mathrm{CI}$ & & 4.0 to 6.0 & 1.9 to 3.4 & 0.4 to 1.2 & 0.9 to 2.0 & 8.6 to 11.3 & & 1.1 to 2.2 & 2.7 to 4.3 & 0.1 to 0.7 & 1.2 to 2.4 & 6.2 to 8.4 \\
\hline
\end{tabular}

CI, confidence interval; MI, myocardial infarction; PVD, peripheral vascular disease; TC, total cholesterol.

\section{Results}

FEATURES OF THE POPULATION

The 3963 subjects with a cholesterol measurement had a mean age of 49 years and a mean systolic blood pressure $132 \mathrm{~mm} \mathrm{Hg}$; $36 \%$ were smokers and $2.3 \%$ had diabetes. The mean serum cholesterol was $6.1 \mathrm{mmol} / 1$ in men and $6.0 \mathrm{mmol} / 1$ in women. Table 1 shows mean data for those with $(\mathrm{n}=3963)$ and without ( $\mathrm{n}=946)$ a cholesterol measurement. Those with no cholesterol measurement were more often women; had a higher prevalence of myocardial infarction, angina, stroke, and peripheral vascular disease; were older; were more often smokers; and had higher blood pressure.

\section{SECONDARY PREVENTION}

Myocardial infarction

The prevalence of a history of myocardial infarction with serum cholesterol $\geqslant 5.0 \mathrm{mmol} /$ 1 was $4.5 \%$ in men, $1.5 \%$ in women, and $2.9 \%$ (95\% CI $2.3 \%$ to $3.4 \%$ ) overall. Removing the $5.0 \mathrm{mmol} / 1 \mathrm{cholesterol}$ threshold increased the prevalence of myocardial infarction to $5.0 \%$ in men, $1.6 \%$ in women, and $3.2 \%$ (95\% CI $2.6 \%$ to $3.7 \%$ ) overall. The increasing prevalence with age is shown in table 2 .

\section{Angina}

The prevalence of self reported angina (but no myocardial infarction) and total cholesterol $\geqslant 5.0 \mathrm{mmol} / 1$ (table 2) rose in men from $0 \%$ at age $35-44$ years to $5.6 \%$ at age $55-64$ years, and in women from $0.9 \%$ at age $35-44$ years to $7.7 \%$ at age $55-64$ years. The overall prevalence was $2.4 \%$ in men, $3.3 \%$ in women, and $2.9 \%(95 \%$ CI $2.3 \%$ to $3.4 \%)$ in men and women together. Removing the $5.0 \mathrm{mmol} / \mathrm{l}$ threshold increased the prevalence from $2.9 \%$ to $3.1 \%$ (95\% CI $2.6 \%$ to $3.6 \%)$, comprising $2.6 \%$ of men and $3.5 \%$ of women.
Stroke

The prevalence of a history of stroke (but no myocardial infarction or angina) and cholesterol $\geqslant 5.0 \mathrm{mmol} / 1$ (table 2 ) was $0.8 \%$ for men, $0.4 \%$ for women, and $0.6 \%(95 \%$ CI $0.3 \%$ to $0.8 \%$ ) overall. No men and only one woman with stroke had a serum cholesterol concentration $<5.0 \mathrm{mmol} / 1$ and therefore the prevalence of stroke was similar when those with cholesterol $<5.0 \mathrm{mmol} / 1$ were included.

\section{Peripheral vascular disease}

The prevalence of peripheral vascular disease and cholesterol $\geqslant 5.0 \mathrm{mmol} / 1$ was $1.3 \%$ in men, $1.7 \%$ in women, and $1.5 \%$ (95\% CI 1.1\% to $1.9 \%$ ) overall. Removing the $5.0 \mathrm{mmol} / \mathrm{l}$ cholesterol threshold increased the overall prevalence from $1.3 \%$ to $1.5 \%$ in men, from $1.7 \%$ to $1.8 \%$ in women, and from $1.5 \%$ to $1.7 \%$ (95\% CI $1.3 \%$ to $2.1 \%$ ) in men and women together.

\section{Myocardial infarction, angina, stroke or} peripheral vascular disease

Combining these indications for secondary prevention, $8.9 \%$ (95\% CI $7.6 \%$ to $10.3 \%$ ) of men, $6.8 \%$ (95\% CI $5.7 \%$ to $7.9 \%$ ) of women, and $7.8 \%$ (95\% CI $6.9 \%$ to $8.6 \%)$ overall gave a history of one of these diagnoses and had serum cholesterol $\geqslant 5.0 \mathrm{mmol} / 1$ (table 2). Removing the cholesterol threshold increased the prevalence to $9.9 \%$ (95\% CI $8.6 \%$ to $11.3 \%$ ) for men, $7.3 \%$ (95\% CI $6.2 \%$ to $8.4 \%$ ) for women, and $8.5 \%$ (95\% CI $7.7 \%$ to $9.4 \%$ ) in all subjects.

\section{PRIMARY PREVENTION}

Tables 3 and 4 show that the CHD risk threshold selected for treatment has a profound effect on the population need for primary prevention.

Table 3 Proportion (\%) of people at different ages with serum total cholesterol $\geqslant 5.0$ mmol/l, and separately for those with any cholesterol concentration who have a predicted risk of CHD events of $30 \%, 20 \%, 15 \%$, or $6 \%$ over 10 years

\begin{tabular}{|c|c|c|c|c|c|c|c|c|c|c|}
\hline \multirow[b]{2}{*}{ Age (years) } & \multirow[b]{2}{*}{ Denominator } & \multicolumn{4}{|c|}{ Men: 10 year risk } & \multirow[b]{2}{*}{ Denominator } & \multicolumn{4}{|c|}{ Women: 10 year risk } \\
\hline & & $\geqslant 30 \%$ & $\geqslant 20 \%$ & $\geqslant 15 \%$ & $\geqslant 6 \%$ & & $\geqslant 30 \%$ & $\geqslant 20 \%$ & $\geqslant 15 \%$ & $\geqslant 6 \%$ \\
\hline \multicolumn{11}{|c|}{$\mathrm{TC} \geqslant 5.0 \mathrm{mmol} / 1$} \\
\hline $35-44$ & 671 & 0.3 & 0.3 & 1.7 & 20.4 & 804 & 0.0 & 0.1 & 0.1 & 1.8 \\
\hline $45-54$ & 583 & 1.0 & 1.0 & 15.3 & 56.0 & 673 & 0.3 & 1.0 & 2.7 & 19.8 \\
\hline $55-64$ & 558 & 7.6 & 7.6 & 35.4 & 61.8 & 674 & 1.4 & 3.9 & 10.8 & 53.7 \\
\hline Total 35-64 & 1812 & 2.8 & 10.1 & 16.3 & 44.4 & 2151 & 0.5 & 1.6 & 4.3 & 23.5 \\
\hline $95 \%$ CI & & 2.0 to 3.5 & 8.7 to 11.4 & 14.6 to 18.0 & 42.1 to 46.7 & & 0.2 to 0.8 & 1.1 to 2.1 & 3.4 to 5.1 & 21.7 to 25.3 \\
\hline \multicolumn{11}{|l|}{ All TC } \\
\hline Total 35-64 & 1812 & 2.9 & 10.9 & 18.1 & 51.3 & 2151 & 0.5 & 1.6 & 4.4 & 25.0 \\
\hline $95 \%$ CI & & 2.1 to 3.6 & 9.5 to 12.4 & 16.3 to 19.8 & 49.0 to 53.6 & & 0.2 to 0.9 & 1.1 to 2.2 & 3.5 to 5.3 & 23.1 to 26.8 \\
\hline
\end{tabular}


Table 4 Proportion (\% and 95\% CI) of the population aged 35-64 years who have serum total cholesterol concentration $\geqslant 5.0 \mathrm{mmol} / \mathrm{l},<5.0 \mathrm{mmol} / \mathrm{l}$, and any total cholesterol

\begin{tabular}{|c|c|c|c|c|}
\hline & Policy & $T C \geqslant 5 \mathrm{mmol} / \mathrm{l}$ & Any TC & $T C<5 \mathrm{mmol} / \mathrm{l}$ \\
\hline & Secondary prevention & $7.8(6.9 \text { to } 8.6)^{\star}$ & $8.5(7.7$ to 9.4$)$ & $0.8(0.5$ to 1.0$)$ \\
\hline and & Primary prevention at $30 \% \dagger$ & $1.5(1.2$ to 1.9$)$ & $1.6(1.2$ to 2.0$)$ & $0.0(0.0$ to 0.1$)$ \\
\hline or & Primary prevention at $20 \%$ & $5.4(4.7$ to 6.1$)$ & $5.8(5.1$ to 6.5$)$ & $0.4(0.2$ to 0.6$)$ \\
\hline or & Primary prevention at $15 \%$ & $9.7(8.8$ to 10.6$)$ & $10.5(9.5$ to 11.5$)$ & $0.8(0.6$ to 1.1$)$ \\
\hline or & Primary prevention at $6 \%$ & $32.9(31.5$ to 34.4$)$ & $36.8(35.3$ to 38.3$)$ & $3.8(3.2$ to 4.4$)$ \\
\hline or & Primary prevention at projected $20 \% \neq$ & $12.4(11.4$ to 13.5$)$ & $13.4(12.3$ to 14.4$)$ & $0.9(0.6$ to 1.2$)$ \\
\hline
\end{tabular}

^Figures are percentages of Scottish men and women age 35-64 years with $95 \%$ CIs.

$\dagger$ Percentages for primary prevention represent CHD risk over 10 years.

$\ddagger$ Risk calculated with age projected to 60 years if current age $<60$.

Treatment at CHD risk $30 \%$ over 10 years targets $2.8 \%$ of all men and $0.5 \%$ of all women, whereas treatment at $6 \%$ over 10 years would embrace $44 \%$ of all men and $24 \%$ of all women. For the whole population aged 35-64 the prevalence of 10 year CHD risk $30 \%$ is $1.5 \%$, of CHD risk $20 \%$ is $5.4 \%$, of $\mathrm{CHD}$ risk $15 \%$ is $9.7 \%$, and of CHD risk $6 \%$ is $32.9 \%$. In practice the proportion of the population for primary prevention would be added to those for secondary prevention, who have top priority. Figure 3 illustrates the need for secondary prevention plus primary prevention at different CHD risk thresholds.

Age has a powerful effect on CHD risk, and the effect of age on population prevalence is most pronounced at lower CHD thresholds. For example, in men the prevalence of $30 \% 10$ year risk increased from $0.3 \%$ at age $35-44$ years to $7.6 \%$ at age $55-64$ years, an increase of 7.3 percentage points. The prevalence of $6 \%$

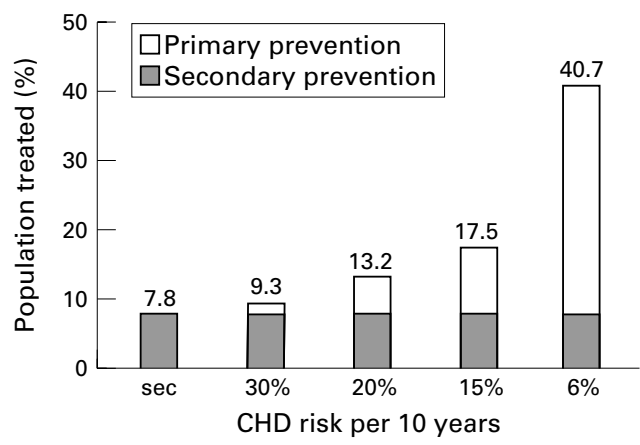

Figure 3 Proportion of the population aged 35-64 years with serum total cholesterol concentration $\geqslant 5.0 \mathrm{mmol} / \mathrm{l}$ for secondary prevention and primary prevention with predicted risk of $C H D$ events of $30 \%, 20 \%, 15 \%$, or $6 \%$ over 10 years.

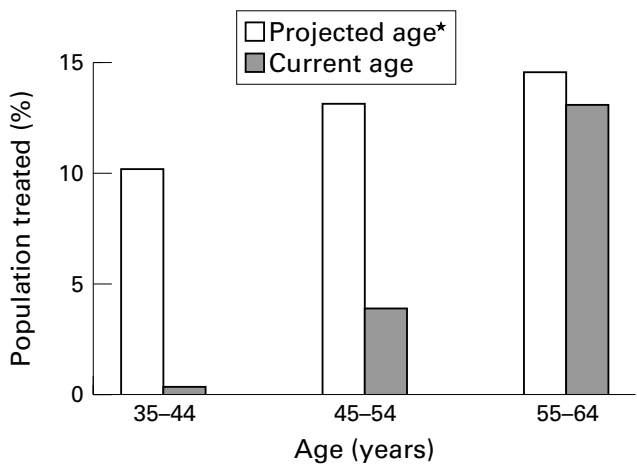

Figure 4 Proportion of people with serum cholesterol concentration $\geqslant 5.0 \mathrm{mmol} / \mathrm{l}$ and $\mathrm{CHD}$ risk $20 \%$ over 10 years using current age, and age projected to age 60 years if current age is $<60^{\star}$ as in the Foint European Task Force guidelines, displayed by age.
10 year CHD risk increased from $20 \%$ at age 35-44 years to $62 \%$ at age $55-64$ years, an increase of 42 percentage points.

At any risk threshold more men than women would need statin treatment, but the sex difference is less pronounced at low risk thresholds and in older people. For example, at age 55-64 years and CHD risk threshold of $6 \%$ over 10 years, $54 \%$ of women and $62 \%$ of men have this risk.

CHOLESTEROL THRESHOLD

Tables 3 and 4 show the effect of removing the $5.0 \mathrm{mmol} / 1 \mathrm{cholesterol}$ threshold. For secondary prevention, the percentage of the population for treatment would increase from $7.8 \%$ to $8.5 \%$. For primary prevention at high CHD risk, the cholesterol threshold makes little discernible difference. As CHD risk is lowered there is some increase in prevalence, for example, from $32.9 \%$ to $36.8 \%$ at the $6 \% 10$ year risk threshold.

\section{PROJECTED CHD RISK}

Projected CHD risk is calculated using the age of 60 years when the actual age is less than 60 years. Figure 4 shows the effect of targeting treatment at actual CHD risk $20 \%$ over 10 years and projected CHD risk 20\% over 10 years. At age 35-44 the population prevalence for the $20 \%$ threshold is $10.2 \%$ for projected risk compared with $0.4 \%$ for actual CHD risk. At age 55-64 the prevalence for projected risk is $14.5 \%$ and for actual risk $13.0 \%$. The overall prevalence for projected CHD risk of $20 \%$ is $12.4 \%(24.1 \%$ of men and $2.9 \%$ of women) and for actual CHD risk $5.4 \%$ (10.1\% of men and $1.6 \%$ of women).

\section{Discussion}

The sample surveyed is closely representative of the Scottish population, ${ }^{15}$ and most had all the data required to assess the need for statin treatment for secondary and primary prevention. Possible sources of bias and error in such data have been discussed in detail elsewhere. ${ }^{7}$ Those who decline to participate generally have higher cardiovascular risk. Those excluded because they had no cholesterol measurement more often reported myocardial infarction, angina, stroke, and peripheral vascular disease (table 1). For primary prevention, those with cholesterol measurement probably had a slightly lower CHD risk than those without cholesterol measurement because of their lower age (by 0.7 years), systolic blood pressure (by $2.9 \mathrm{~mm} \mathrm{Hg}$ ), prevalence of smoking (by 
9.6\%), and prevalence of diabetes (by $1.6 \%$ ). The need for statin treatment in the whole population may therefore be underestimated. Other sources of bias or error include use of self reported diagnoses; inclusion of cerebral haemorrhage or embolic stroke from the heart in the all encompassing diagnosis of "stroke"; and the use of only one measurement of serum total cholesterol and HDL cholesterol. Despite these shortcomings the population estimates obtained from this type of analysis are likely to be reasonably accurate, as we have discussed elsewhere. ${ }^{7}$

IMPLICATIONS FOR STATIN TREATMENT

Secondary prevention

About $7.8 \% \quad(8.9 \%$ of men and $6.8 \%$ of women) of the Scottish population aged 35-64 years would need secondary prevention with a statin using cholesterol $\geqslant 5.0 \mathrm{mmol} / 1$ as threshold. Removing the $5.0 \mathrm{mmol} / \mathrm{l}$ cholesterol threshold for treatment would increase the need for statins from $7.8 \%$ to $8.5 \%$ of people. These figures considerably underestimate the total need for secondary prevention in the population because there is evidence for benefit up to the age of 75 years, ${ }^{2}$ and the upper age in this survey was 64 years. Implementation of secondary prevention alone is a major undertaking.

\section{Primary prevention}

The CHD risk targeted for primary prevention determines the number needed to treat, ${ }^{9}$ cost effectiveness, ${ }^{10}$ proportion of the population requiring treatment, ${ }^{7}$ and the total cost of treatment. ${ }^{70}$ This analysis sets out the population implications of treatment policies at four levels of CHD risk. Diet and lifestyle measures would be advised before statin treatment for primary prevention, but their effect on CHD risk in controlled trials is modest. ${ }^{19-21}$ Statin treatment can be justified at CHD risk $\geqslant 6 \%$ over 10 years, which was the risk of placebo treated patients in the AFCAPS/TexCAPS study. ${ }^{5}$ This would entail treating $33 \%$ of the Scottish population aged 35-64 (44\% men, $24 \%$ women) for primary prevention. Adding the $7.8 \%$ of people who need secondary prevention, no less than $41 \%$ of all adults aged 35-64 would be treated with statins. This is beyond any health care system on grounds of both cost and workload, and because of this recent Joint British guidelines have recommended treatment at a higher CHD risk threshold. ${ }^{11}$

Treatment for primary prevention at a CHD risk of $30 \%$ over 10 years with total cholesterol concentration $\geqslant 5.0 \mathrm{mmol} / 1$, recommended as a minimum in these guidelines, would entail statin treatment of $1.5 \%$ of the population aged 35-64 for primary prevention and of $9.3 \%$ in total for secondary and primary prevention. The Joint British guidelines also recommend a progressive move to treatment at $15 \%$ over 10 years as resources permit. ${ }^{11}$ This would entail statin treatment for $9.7 \%$ of people for primary prevention and $17.5 \%$ for primary plus secondary prevention. This can not be implemented in the UK at present because of the cost and workload but may be feasible in populations with lower mean cholesterol values, a lower absolute CHD risk in relation to cholesterol, or both.

EFFECT OF THE CHOLESTEROL THRESHOLD Guidelines currently advise treatment at a cholesterol threshold of $5.0 \mathrm{mmol} / \mathrm{l}$ based on a subgroup analysis of the CARE (cholesterol and recurrent events) trial, ${ }^{2}$ and it remains possible that statins should be prescribed for all those with high CHD risk regardless of the total cholesterol concentration. There are suggestions that all patients who have survived a myocardial infarction should have statin treatment. Table 4 shows the likely increase in statin treatment if the $5.0 \mathrm{mmol} / \mathrm{l} \mathrm{cholesterol} \mathrm{thresh-}$ old were removed. The percentage of the population aged 35-64 years needing treatment for secondary prevention would rise from $7.8 \%$ to $8.5 \%$. For primary prevention, removal of the cholesterol threshold makes little difference at high CHD risk. The percentage of the population with $\mathrm{CHD}$ risk $30 \%$ over 10 years is $1.5 \%$ with cholesterol $\geqslant 5.0 \mathrm{mmol} / 1$ and $1.6 \%$ when the threshold is removed, implying that high cholesterol is generally required to attain this level of risk. At low CHD risk, the percentage increase is greater. For example the prevalence of CHD risk $6 \%$ per 10 years increases from $33 \%$ to $37 \%$ when the $5.0 \mathrm{mmol} / 1$ cholesterol threshold is disregarded.

PROJECTED CHD RISK

British guidelines recommend that statin treatment be targeted at absolute coronary risk, ${ }^{11}$ whereas Joint European Task Force guidelines advise statin treatment for "projected risk" defined as the absolute risk of a person using age 60 years when the current age is less than 60 years. ${ }^{12}$ This policy increases statin treatment of young people massively (fig 4 ). No less than $21.7 \%$ of men aged $35-39$ would be treated using age projected to 60 , whereas none would be treated using actual age. The average CHD risk of people in the Scottish health survey aged $35-39$ is $3.0 \%$ over 10 years: $4.9 \%$ in men and $1.2 \%$ in women. The average CHD risk in AFCAPS/TexCAPS was higher than this, at $6 \%$ over 10 years. ${ }^{5}$ Treatment aimed at projected risk would therefore entail treatment of over one fifth of men aged 35-39 who have a level of risk that has not been examined in randomised controlled trials with statins. At this CHD risk, 200 people would need treatment for five years to prevent one event at a cost of $£ 556000$ for each event prevented.

COMPARISON WITH ANALYSIS OF HEALTH SURVEY FOR ENGLAND

We have previously reported the implications of targeting statin treatment at different CHD risk thresholds in the English population. ${ }^{7}$ The present analysis in the Scottish population has similarities but is not directly comparable. Age in the Scottish population sample was 35-64 years and in the English sample 35-69 years; peripheral vascular disease was not elicited in the English survey, thus underestimating the 
need for secondary prevention; and the English survey did not measure HDL cholesterol, so that risk prediction for primary prevention was less accurate. The last difference is important because the average HDL in the Scottish sample $(1.30 \mathrm{mmol} / 1$ for men and $1.56 \mathrm{mmol} / 1$ for women) was, surprisingly, much higher than the values from published sources assumed in our analysis of the English population (1.15 for men and 1.40 for women). ${ }^{7}$ Direct comparisons between the two analyses are therefore difficult. However, this study suggests a higher need for secondary prevention in the Scottish population $(7.8 \% v 4.8 \%$ in the English population), and this difference would be even more pronounced if the age range in the Scottish population were extended to 69 years. Primary prevention for subjects with a CHD event rate of $30 \%$ over 10 years was predicted for $3.4 \%$ of the English population aged 35-69, compared with $1.5 \%$ of the Scottish population aged 35-64. At CHD risk 15\% over 10 years, $19.6 \%$ of the English population and $9.7 \%$ of the Scottish population appeared to be candidates for treatment. The apparent need for more primary prevention in the English population is likely an artefact due to the older age in the English sample, the lower HDL assumed, and the higher cholesterol threshold $(5.5 \mathrm{mmol} / \mathrm{l})$ that was appropriate then, compared with $5.0 \mathrm{mmol} / 1$ now.

We thank the original depositors and data archive for access to data from the Scottish health survey 1995. Those who conducted the survey and the original analysis of the data bear no responsibility for their further analysis or interpretation. We no responsibility for their further analysis or interpretation. We sion of the controller of HMSO. Conflict of interest: none.

1 Scandinavian Simvastatin Survival Study Group. Randomised trial of cholesterol lowering in 4444 patients with CHD: the Scandinavian simvastatin survival study (4S). Lancet 1994;344:1383-9.

2 Sacks FM, Pfeffer MA, Moye LA, et al. The effect of pravastatin on coronary events after myocardial infarction in patients with average cholesterol levels. N Engl f Med 1996; 335:1001-9.

3 The Long-term Intervention with Pravastatin in Ischaemic Disease (LIPID) Study Group. Prevention of cardiovascular events and death with pravastatin in patients with coronary heart disease and a broad range of initial cholesterol levels. N Engl F Med 1998;339:1349-57.

4 Shepherd J, Cobbe SM, Ford I, et al. Prevention of coronary heart disease with pravastatin in men with hypercholesterolaemia. N Engl F Med 1995;333:1301-7.

5 Downs JR, Clearfield M, Weis S, et al for the AFCAPS/ TexCAPS Research Group. Primary prevention of acute coronary events with lovastatin in men and women with average cholesterol levels. $7 A M A$ 1998;279:1615-22.

6 Glasziou PP, Irwig LM. An evidence based approach to individualising patient treatment. BMF 1995;311:1356-9.

7 Haq IU, Ramsay LE, Pickin DM, et al. Lipid-lowering for prevention of coronary heart disease: what policy now? Clin Sci 1996;91:399-413.

8 Ramsay LE, Haq IU, Jackson PR, et al. The Sheffield table for primary prevention of coronary heart disease: corrected. Lancet 1996;348:1251-2.

9 Cook RJ, Sackett DL. The number needed to treat: a clinically useful measure of treatment effect. BMF 1995;310: $452-4$.

10 Pickin DM, McCabe CJ, Ramsay LE, et al. Costeffectiveness of HMG CoA reductase inhibitor (statin) treatment related to the risk of coronary heart disease and cost of drug treatment. Heart 1999;82:325-32.

11 Wood D, Durrington P, Poulter N, et al on behalf of the British Cardiac Society, British Hyperlipidaemia Association, British Hypertension Society and endorsed by the British Diabetic Association. Joint British recommen tions on prevention of coronary heart disease in clinical practice. Heart 1998;80 (Suppl 2):S1-29.

12 Wood D, De Backer G, Faergeman O, et al. Prevention of coronary heart disease in clinical practice. Recommendations of the Second Joint Task Force of European and other societies on coronary prevention. Eur Heart f 1998;19: 1434-503.

13 Standing Medical Advisory Committee. The use of statins (11061 HCD Aug 97(04)). London: Department of Health, 1997.

14 Scottish Intercollegiate Guidelines Network (SIGN). Lipids and the primary prevention of coronary heart disease. Edinburgh: SIGN, 1998.

15 Joint Health Surveys Unit of Social and Community Planning Research and University College London. Scottish health survey, 1995, 2nd edn (SN;3807) [computer program]. Colchester:. The Data Archive (distributor), 30th November 1998.

16 Haq IU, Ramsay LE, Jackson PR, et al. Prediction of coronary risk for primary prevention of coronary heart disease: a comparison of methods. $Q \mathcal{F}$ Med 1999;92:379-85.

17 Durrington PN, Prais H, Bhatnagar D, et al. Indication for Durrington PN, Prais $\mathrm{H}$, Bhatnagar $\mathrm{D}$, et al. Indication for
cholesterol-lowering medication: comparison of riskassessment methods. Lancet 1998;353:278-81.

18 Anderson KM, Odel PM, Wilson PWF, et al. Cardiovascular disease risk profiles. Am Heart f 1991;121:293-8.

19 Ramsay LE, Yeo WW, Jackson PR. Dietary reduction if serum cholesterol concentration: time to think again. BMF 1991;303:953-7.

20 Tang JL, Armitage JM, Lancaster T, et al. Systematic review of dietary intervention trials to lower blood total cholesterol of dietary intervention trials to lower blood total
in free-living subjects. BMF 1998;316:1213-20.

21 Ebrahim S, Davey Smith G. Systematic review of randomised controlled trials of multiple risk factor interventions for preventing coronary heart disease. BMF 1997;314: 1666-74 